\title{
Evidence from Tm anomalies for non-CI refractory lithophile element proportions in terrestrial planets and achondrites
}

\author{
by
}

\author{
J.A. Barrat ${ }^{1}$, N. Dauphas ${ }^{2}$, P. Gillet ${ }^{3}$, C. Bollinger ${ }^{4}$, \\ J. Etoubleau ${ }^{5}$, A. Bischoff ${ }^{6}$, and A. Yamaguchi ${ }^{7}$
}

1: Université de Bretagne Occidentale, Institut Universitaire Européen de la Mer, CNRS UMR 6538, Place Nicolas Copernic, 29280 Plouzané, France. E-mail: barrat@univ-brest.fr

2: Origins Laboratory, Department of the Geophysical Sciences and Enrico Fermi Institute, The University of Chicago, 5734 South Ellis Avenue, Chicago IL 60637, USA

3: Ecole Polytechnique Fédérale de Lausanne (EPFL), Institute of Condensed Matter Physics, Station 3, CH-1015 Lausanne, Switzerland.

4: Institut Universitaire Européen de la Mer, CNRS UMS 3113, Place Nicolas Copernic, 29280 Plouzané Cedex, France.

5: IFREMER, centre de Brest, 29280 Plouzané, France.

6: Institut für Planetologie, Westfälische Wilhelms-Universität Münster, Wilhelm-Klemm-Str. 10, 48149 Münster, Germany.

7: National Institute of Polar Research, Tachikawa, Tokyo 190-8518, Japan and Department of Polar Science, School of Multidisciplinary Science, SOKENDAI (The Graduate University for Advanced Studies), Tachikawa, Tokyo 190-8518, Japan

Key words: Rare Earth Elements (REE), achondrite, Earth, Mars, Tm anomaly.

Submitted to Geochimica Cosmochimica Acta, 6/25/15

Revised version 11/29/15 


\begin{abstract}
Thulium is a heavy rare earth element (REE) whose geochemical behavior is intermediate between $\mathrm{Er}$ and $\mathrm{Yb}$, and that is not expected to be decoupled from these elements during accretion of planetary bodies and geological processes. However, irregularities in REE volatilities at higher temperature could have decoupled the REEs relative to one another during the early stages of condensation of the solar nebula. Indeed, positive Tm anomalies are found in some refractory inclusions from carbonaceous chondrites, and it is possible that large scale nebular reservoirs displaying positive or negative Tm anomalies were formed during the early history of the solar system. We analyzed a series of meteorites and terrestrial rocks in order to evaluate the existence of $\mathrm{Tm}$ anomalies in planetary materials. Relative to CIs (Ivuna-type carbonaceous chondrites), carbonaceous chondrites display unresolved or positive $\mathrm{Tm}$ anomalies, while most of the noncarbonaceous chondrites show slightly negative $\mathrm{Tm}$ anomalies. Quantification of these anomalies in terrestrial samples is complicated when samples display fractionated heavy REE patterns. Taking this effect into account, we show that the Earth, Mars, Vesta, the aubrite and ureilite parent bodies display small negative anomalies $(\mathrm{Tm} / \mathrm{Tm} * \approx 0.975)$, very similar to those found in ordinary and enstatite chondrites. We suggest that a slight negative Tm anomaly relative to $\mathrm{CI}$ is a widespread feature of the materials from the inner solar system. This finding suggests that CI chondrites may not be appropriate for normalizing REE abundance patterns of most planetary materials as they may be enriched in a hightemperature refractory component with non-solar composition. The presence of $\mathrm{Tm}$ anomalies at a bulk planetary scale is, to this day, the strongest piece of evidence that refractory lithophile elements are not present in constant proportions in planetary bodies.
\end{abstract}

\title{
1. Introduction
}

Rare Earth Elements (REEs) are a group of elements whose geochemical properties are very similar to each other. They are generally trivalent with ionic radii decreasing smoothly from La to $\mathrm{Lu}$. The coherence of their behaviors makes them prime elements for geochemical modeling. Their abundances are classically shown with "Masuda-Coryell plots", where the ratio of each REE concentration to the corresponding value in a reference is plotted as a function of atomic number (Masuda, 1962; Coryell et al., 1963). The references most commonly used in this normalization scheme are a CI chondrite average (e.g., Anders and Grevesse, 1989; Pourmand et al., 2012; Barrat et al., 2012; Palme et al., 2014) and post Archean Australian shale (PAAS; e.g., Nance and Taylor, 1976; Taylor and McLennan, 1985; Pourmand et al., 2012). The resulting curves, known as REE patterns, became one of the most widely used tools to trace geological processes (e.g., Frey et al., 1978; Hanson, 1980; Taylor and McLennan, 1981). These diagrams provide a means of assessing the REE abundances of many samples at a glance, and to detect easily the decoupling of some elements relative 
to their neighbors, when the conditions that prevail in the system compel them to a different valence. On Earth for example, Eu and $\mathrm{Ce}$ anomalies are found in many magmatic rocks and in aqueous surface environments, respectively when $\mathrm{Eu}$ and $\mathrm{Ce}$ are present in their $2+$ or $4+$ valence states while other lanthanides are in their $3+$ valence state.

REEs are refractory elements, meaning that their temperatures of condensation during cooling of a solar gas composition at conditions relevant to the formation of the solar system are higher than those of major elements such as Mg or Si (e.g., Boynton, 1975; Davis and Grossman, 1979, Lodders, 2003). Their temperatures of condensation vary from one REE to another, so they can be fractionated from each other at high temperature during evaporation or condensation processes (e.g., Pack et al., 2004 and references therein). This is best illustrated with refractory inclusions found in chondrites, which are the first solids formed in the solar system, that display distinctive volatility-controlled REE patterns with a variety of $\mathrm{Ce}, \mathrm{Sm}, \mathrm{Eu}, \mathrm{Tm}$ and $\mathrm{Yb}$ anomalies (e.g., Tanaka and Masuda, 1973; Mason and Taylor, 1982; MacPherson, 2003; Fegley and Ireland, 1991; Hiyagon et al., 2011 and references therein).

Inductively Coupled Plasma-Mass Spectrometry (ICP-MS) is established as a premier technique for the determination of trace element abundances in rocks. Unlike previous analytical procedures such as neutron activation or isotope dilution-thermo-ionization mass spectrometry, ICPMS can simultaneously measure the concentrations of all the REEs present in a given sample. Thus, ICP-MS can allow us to detect small anomalies that were previously not accessible using earlier techniques.

Until recently, it was thought that the REE patterns of terrestrial rocks could only display Eu or $\mathrm{Ce}$ anomalies. Thus, the discovery of slight negative $\mathrm{Tm}$ anomalies in shales when normalized to CI chondrite was surprising (Pourmand et al., 2012). This observation was subsequently confirmed in some other terrestrial samples and extended to ordinary chondrites, enstatite chondrites, samples from Mars, Moon and Vesta (Bendel et al. 2011, 2012a,b; Dauphas and Pourmand, 2015). Because the negative Tm anomalies obtained in planetary materials by previous workers are small (a few percent), one may argue that they could be at the limit of the precision of the analytical methods, or artifacts produced by an overestimation of the Tm abundance in the chondritic reference. In this paper, we report on analyses obtained on 87 terrestrial and meteoritic samples using a well-established ICP-MS procedure (e.g., Barrat et al., 2012). Our aim is firstly to confirm the Tm abundance in average CI chondrites, secondly to evaluate the diversity of Tm anomalies in differentiated planetary bodies such as Mars and Vesta, which were not investigated in the recent study of Dauphas and Pourmand (2015), which focused on chondrites and Earth, and finally to determine the Tm anomaly of the bulk Earth. 


\section{Samples and analytical methods}

Seventeen chondrites, seven eucrites (from asteroid 4 Vesta), six shergottites (from Mars), one lunar meteorite, nine other achondrites (ureilites, aubrites, one angrite and one ungrouped achondrite), forty-six samples from the Earth (including reference materials), and one olivine fraction from a pallasite, were selected in order to capture the diversity of chondrites and magmatic rocks from Earth, Mars and asteroids. These samples were obtained during the last twenty-five years from various sources including the Muséum National d'Histoire Naturelle de Paris (MNHN), Ifremer (Plouzané, France), Université de Rennes 1 (France), Université de Paris VI (France), Université de Bretagne Occidentale (Brest), the National Institute of Polar research (Tokyo), the Natural History Museum, Vienna, the Smithsonian Institution, the Meteorite Working Group (NASA), the Institute for Meteoritics, Albuquerque, the Northern Arizona University, Flagstaff, and meteorite collectors. The details of the meteorite samples used in this study are given in supplementary Table S1.

Most of the samples were previously characterized extensively for their chemical and mineralogical compositions. The concentrations of some REEs were previously determined in various laboratories using different analytical techniques but with a few exceptions, mono-isotopic Tm was not analyzed. In this study, REE abundances were determined using a Thermo Element 2 ICP-mass spectrometer at Institut Universitaire Européen de la Mer (IUEM), Plouzané, following a wellestablished procedure first developed at the University of Southampton and Université Joseph Fourier (Barrat et al., 1996), and regularly improved since then (e.g., Barrat et al., 2012). The methodology is briefly summarized below.

Samples were finely powdered using a boron carbide mortar and pestle. Typically, $120 \mathrm{mg}$ of the powder were dissolved in closed screw-top teflon vessels (Savillex) at about $130{ }^{\circ} \mathrm{C}$ for three days using $5 \mathrm{ml}$ of concentrated $\mathrm{HF}$ and $2 \mathrm{ml}$ of concentrated $\mathrm{HNO}_{3}$. The vessels were then opened. After evaporation to dryness of the acid mixture, approximately $2 \mathrm{ml}$ of $\mathrm{HNO}_{3}$ was added, and the vessels were capped and put back on the hot plate and left overnight. The samples were then dried again, and taken up in about $20 \mathrm{~g}$ of $6 \mathrm{M} \mathrm{HCl}$ ("mother solutions"). No residual grains were observed in the mother solutions except for ureilites, which contained some carbon grains (low REE concentration graphite and diamond) having no impact on the results. Only reagents double-distilled in quartz or teflon sub-boilers were used. For each sample, the abundances of REEs were determined using two aliquots of the mother solution.

A first aliquot of the mother solution was dried and the residue was taken up and dissolved in diluted $\mathrm{HNO}_{3}(2 \%)$ containing traces of HF. This sample solution (solution 1) was analyzed in triplicate, in a sequence containing a procedural blank, a BHVO-2 reference solution (prepared like the 
samples), and a series of solutions for the corrections of oxide and hydroxide interferences (pure water, $\mathrm{Ba}+\mathrm{Ce}, \mathrm{Pr}+\mathrm{Nd}$, and $\mathrm{Sm}+\mathrm{Eu}+\mathrm{Gd}+\mathrm{Tb}$ solution, see Barrat et al. (1996) for more details). One BHVO-2 reference solution was analyzed every three samples and used for both calibration and drift corrections. The raw data were first corrected for drift, procedural blank and interferences. $[\mathrm{X}]_{1}$, the raw concentration of a given element $\mathrm{X}$ in sample was calculated using the corrected data for the BHVO-2 and sample solutions. Then, the three analyses obtained for each sample were averaged.

A second aliquot of the mother solution was spiked with a pure solution of Tm, and processed like the previous one. The spiked sample solution (solution 2) was analyzed using a separate sequence similar to that used for unspiked samples. The REE abundances (except Tm) in the sample are calculated with the following equation (Barrat et al., 1996):

$$
[\mathrm{X}]_{2}=\left(\mathrm{C}_{\mathrm{X}} / \mathrm{C}^{\prime}{ }_{\mathrm{Tm} 1}\right)\left(\mathrm{M}_{\mathrm{Tm}} / \mathrm{M}\right) /\left[\left(\mathrm{C}_{\mathrm{Tm}} / \mathrm{C}^{\prime}{ }_{\mathrm{Tm} 1}\right)-1\right]
$$

Where $[\mathrm{X}]_{2}$ and $\mathrm{C}_{\mathrm{x}}$ are the concentrations of the element $\mathrm{X}$ in the sample and in the solution 2 , respectively. $\mathrm{C}_{\mathrm{Tm}}$ and $\mathrm{C}^{\prime}{ }_{\mathrm{Tm} 1}$ are respectively the concentration of $\mathrm{Tm}$ in solution 2 and the estimated concentration of $\mathrm{Tm}$ in this solution without the contribution of the spike. $\mathrm{C}^{\prime}{ }_{\mathrm{Tm} 1}$ was directly calculated using the $\mathrm{Tm} / \mathrm{Er}$ and $\mathrm{Tm} / \mathrm{Yb}$ ratios previously obtained for the unspiked solution (solution 1). $\mathrm{M}$ is the mass of sample contained in the aliquot of the mother solution used to prepare solution 2, and $\mathrm{M}_{\mathrm{Tm}}$ is the amount of Tm added. Note that the choice of a Tm-spike here is not specific to the present study but corresponds to the routine procedure used by the first author in Brest.

REE abundances obtained with solutions 1 and 2 are of course very similar, but Tm abundances cannot be determined using solution 2. However, the concentrations obtained with solution 2 are considered more robust, because less affected by the errors on the dilution factors, and less prone to the instrumental drift (Barrat et al., 1996) than the concentrations calculated with solution 1. On the other hand, REE ratios determined with the unspiked solution (solution 1) are more accurate because the solution was run in triplicate. Therefore, $[X]$, the final REE concentrations were calculated combining $[\mathrm{X}]_{1}$ and $[\mathrm{X}]_{2}$, the results for both solutions as follow:

$$
[\mathrm{X}]=\mathrm{K}[\mathrm{X}]_{1}
$$

where $\quad \mathrm{K}=\left([\mathrm{La}]_{2} /[\mathrm{La}]_{1}+[\mathrm{Ce}]_{2} /[\mathrm{Ce}]_{1}+[\operatorname{Pr}]_{2} /[\mathrm{Pr}]_{1}+[\mathrm{Nd}]_{2} /[\mathrm{Nd}]_{1}+[\mathrm{Sm}]_{2} /[\mathrm{Sm}]_{1}+[\mathrm{Eu}]_{2} /[\mathrm{Eu}]_{1}+[\mathrm{Gd}]_{2} /[\mathrm{Gd}]_{1}+\right.$ $\left.[\mathrm{Tb}]_{2} /[\mathrm{Tb}]_{1}+[\mathrm{Dy}]_{2} /[\mathrm{Dy}]_{1}+[\mathrm{Ho}]_{2} /[\mathrm{Ho}]_{1}+[\mathrm{Er}]_{2} /[\mathrm{Er}]_{1}+[\mathrm{Yb}]_{2} /[\mathrm{Yb}]_{1}+[\mathrm{Lu}]_{2} /[\mathrm{Lu}]_{1}\right) / 13$

Two samples (NWA 7325, and an olivine separate from the Brenham pallasite) display very low heavy REE abundances $(<0.1 \mathrm{x}$ CI). Their REEs were concentrated and separated from the major 
elements before analysis following the method described by Barrat et al. (1996). These two samples were previously analyzed without REE separation and Tm abundances were not available (Greenwood et al., 2015; Barrat et al., 2015). The new analyses now include Tm, and the other REE abundances are in excellent agreement with the previous ones (the previous and new analyses usually agree within $4 \%)$.

The results for international reference materials are given in Table 1, relative to our working values for the USGS basalt BHVO-2. The latter were derived from the isotope dilution results obtained by Raczek et al. (2001) for $\mathrm{La}, \mathrm{Ce}, \mathrm{Nd}, \mathrm{Sm}, \mathrm{Eu}, \mathrm{Gd}$, Dy, Er, Yb and $\mathrm{Lu}$, and from our previous ICP-MS analyses for Pr, Tb, Ho, and Tm (Barrat et al., 2012). In the event of future change to these BHVO-2 values, the data need only to be corrected by the ratio of the new and old values. Based on replicate analyses of standard reference materials and samples (Barrat et al., 2012, 2014), the precision for REE abundances is much better than $3 \%$ (one relative standard deviation - RSD).

The accuracy of our results is of course directly linked to the accuracy of our working values for BHVO-2. At present, BHVO-2 is one of the best characterized reference materials for REEs, and our working values are very similar to the GEOREM's preferred values (georem.mpchmainz.gwdg.de). Furthermore, the results obtained for other well-characterized reference materials (e.g., Bayon et al., 2009; Barrat et al., 2012, 2014) confirm that the BHVO-2 working values are accurate and validate our calibration, as exemplified here by the results obtained for BIR-1 and BCR-2 (Table 1 and Fig. 2), which are other well-known reference materials.

The $\mathrm{Tm}$ anomalies can be estimated using the $\mathrm{Tm} / \mathrm{Tm} *$ ratio, where $\mathrm{Tm} *$ is the interpolated Tm concentration for a smooth CI-normalized REE pattern and $X_{n}$ is the concentration of element $\mathrm{X}$ normalized to chondrite:

$$
\mathrm{Tm} / \mathrm{Tm} *=\left(\operatorname{Tm}_{n}\right) /\left(\mathrm{Er}_{\mathrm{n}} \times \mathrm{Yb}_{\mathrm{n}}\right)^{1 / 2}
$$

The relative standard deviations of $\mathrm{Tm} / \mathrm{Tm}$ * values based on replicate BCR-2 and BIR-1 are better than $0.6 \%$ and $0.3 \%$, respectively (Table 1). From the results obtained for both reference materials and sample replicates, we estimate that the precision of $\mathrm{Tm} / \mathrm{Tm} *$ measurements is better than $0.7 \%$ (1 RSD).

Dauphas and Pourmand (2015) used different equations to estimate the Tm anomalies. In a first equation, $\mathrm{Tm}^{*}$ is interpolated linearly using the logarithm of the CI-normalized abundances against ionic radius of $\mathrm{Er}$ and $\mathrm{Yb}$ : 


$$
\left(\mathrm{Tm} / \mathrm{Tm}^{*}\right)_{\mathrm{D \& P}}=\left(\mathrm{Tm}_{\mathrm{n}}\right) /\left(\mathrm{Er}_{\mathrm{n}}^{0.55} \times \mathrm{Yb}_{\mathrm{n}}^{0.45}\right)
$$

As shown in Figure 1, the Tm anomalies obtained using this equation are similar to those obtained with equation (3). Moreover, Dauphas and Pourmand (2015) proposed a second estimation of the $\mathrm{Tm}$ anomalies using $\mathrm{Er}$ and $\mathrm{Lu}$, because $\mathrm{Yb}$ can be decoupled from the other REEs in highly reducing conditions (e.g., Pack et al., 2004):

$$
(\mathrm{Tm} / \mathrm{Tm} * *)_{\mathrm{D} \& \mathrm{P}}=\left(\mathrm{Tm}_{\mathrm{n}}\right) /\left(\mathrm{Er}_{\mathrm{n}}{ }^{0.66} \times \mathrm{Lu}_{\mathrm{n}}{ }^{0.34}\right)
$$

Finally, they noticed that the curvature of the REE patterns could affect the Tm anomalies when based on simple linear interpolations. They proposed a third approach for normalizing Tm by using a Lagrangian $\left(3^{\text {rd }}\right.$ order polynomial) interpolation between four REEs, Dy, Ho, Er and Lu:

$$
\left(\mathrm{Tm} / \mathrm{Tm} \mathrm{T}^{* * *}\right)_{\mathrm{D \& P}}=\left(\mathrm{Tm}_{\mathrm{n}}\right) /\left(\mathrm{Dy}_{\mathrm{n}}{ }^{0.33} \times \mathrm{Ho}_{\mathrm{n}}^{-1.29} \times \mathrm{Er}_{\mathrm{n}}{ }^{1.85} \times \mathrm{Lu}_{\mathrm{n}}{ }^{0.11}\right)
$$

The enstatite meteorites that we have analyzed do not display negative $\mathrm{Yb}$ anomalies. The $\left(\mathrm{Tm} / \mathrm{Tm}{ }^{* *}\right)_{\mathrm{D \& P}}$ and $\mathrm{Tm} / \mathrm{Tm} *$ ratios are very similar (Fig. 1), and consequently the use of equation 5 is not necessary to compare our enstatite chondrites (EC) samples with the other chondrites. Although the $(\mathrm{Tm} / \mathrm{Tm} * *)_{\mathrm{D} \& \mathrm{P}}$ and $\left(\mathrm{Tm} / \mathrm{Tm} \mathrm{m}^{* *}\right)_{\mathrm{D \& P}}$ ratios have their advantages, they will not be used here. In the rest of the paper, we will use the $\mathrm{Tm} / \mathrm{Tm} *$ ratios calculated using equation 3 because these ratios provide a precise estimate of the Tm-anomalies, are coherent with the inter-element distance in usual REE plots, and provide in most cases, the same information as the $\left(\mathrm{Tm} / \mathrm{Tm}{ }^{*}\right)_{\mathrm{D \& P}}$ and $\left(\mathrm{Tm} / \mathrm{Tm}{ }^{* * *}\right)_{\mathrm{D \& P}}$ ratios. In order to better discuss the curvature effect (CE) of the REE pattern, an additional equation is preferred. We use a Lagrangian interpolation like Dauphas and Pourmand (2015), but with two elements at each side of Tm (Ho, Er, Yb, Lu):

$$
\mathrm{Tm} / \mathrm{Tm}^{*}{ }_{\mathrm{CE}}=\mathrm{Tm}_{\mathrm{n}} /\left[\left(\mathrm{Yb}_{\mathrm{n}} \times \mathrm{Er}_{\mathrm{n}}\right)^{4} /\left(\mathrm{Lu}_{\mathrm{n}} \times \mathrm{Ho}_{\mathrm{n}}\right)\right]^{1 / 6}
$$

As shown in Figure 1, the $\mathrm{Tm} / \mathrm{Tm}{ }_{\mathrm{CE}}$ ratios are more strongly correlated with $\mathrm{Tm} / \mathrm{Tm} *$ than the $(\mathrm{Tm} / \mathrm{Tm} * * *)_{\mathrm{D} \& \mathrm{P}}$ ratios, suggesting that the effect of the shape of the REE pattern is more correctly taken into account with equation 7. The effect of the curvature of the pattern on the calculated $\mathrm{Tm} / \mathrm{Tm} *$ ratio for each sample can be discussed using the CE\# number, defined here as the $\mathrm{Tm}{ }^{*} \mathrm{CE}$ $/ \mathrm{Tm} *$ ratio:

$$
\mathrm{CE} \#=\mathrm{Tm}^{*}{ }_{\mathrm{CE}} / \mathrm{Tm} *=\left[\left(\mathrm{Yb}_{\mathrm{n}} \times \mathrm{Er}_{\mathrm{n}}\right) /\left(\mathrm{Lu}_{\mathrm{n}} \times \mathrm{Ho}_{\mathrm{n}}\right)\right]^{1 / 6}
$$


If $\mathrm{CE} \# \approx 1$ (i.e., in the range $0.99-1.01$ ), the curvature effect is negligible, and the Tm anomaly estimated using equation 3 is reliable. If $\mathrm{CE} \#<1$, the $\mathrm{Tm} / \mathrm{Tm}^{*}$ ratio amplifies the negative $\mathrm{Tm}$ anomalies and reduces the positive ones. If CE\# >1, the opposite effects are obtained. As we will see for terrestrial lavas, the shapes of the REE patterns can have a significant effect on the $\mathrm{Tm} / \mathrm{Tm} *$ ratios. Although, these shapes do not generate anomalous or aberrant Tm anomalies, the curvature effects must be taken into account when the $\mathrm{Tm} / \mathrm{Tm} *$ ratios of samples with very different REE patterns are compared.

The choice of the normalizing values (i.e., CI concentrations) has an important impact on the values of the calculated $\mathrm{Tm} / \mathrm{Tm} *$ ratios. However, it has no effect on the offsets between samples of various types. Here we have adopted the CI average recommended by Barrat et al. (2012) because this average was obtained from five "large" samples prepared from distinct stones of Orgueil, which comprise $c a .4 \mathrm{~g}$ of this meteorite. More importantly, the Orgueil analyses were obtained using the same procedure and the same calibration strategy as the analyses presented here, minimizing any possible systematic bias. We have reanalyzed aliquots of our Orgueil samples (see below), and the new results confirm the results from Barrat et al. (2012), so that revision of the normalizing values is not necessary.

The Tm anomalies reported in this study are small $(<15 \%)$, but significant as illustrated by the reference materials (Table 1): BIR-1 $\left(\mathrm{Tm} / \mathrm{Tm} \mathrm{m}^{*}=0.978,1 \sigma=0.003\right.$, Table 1$)$ and BCR-2 $\left(\mathrm{Tm} / \mathrm{Tm}^{*}=0.970,1 \sigma=0.006\right.$, Table 1$)$ have $\mathrm{Tm} / \mathrm{Tm} *$ ratios that are respectively $7 \sigma$ and $5 \sigma$ below the mean CI value. Comparison with literature results is not straightforward because systematic biases between laboratories are present (Fig. 2). To mitigate this problem, we have adjusted the results obtained by Pourmand et al. (2012, 2014), Stracke et al. (2012), Dauphas and Pourmand (2015) to our calibration using BIR-1, BCR-2, and BHVO-2, and Khan et al. (2015) using the Smithsonian Institution Allende powder (USNM 3529) as follow:

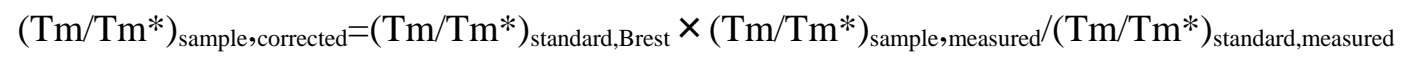

where $\mathrm{Tm}^{*}$ is calculated using the average CI abundances obtained by Barrat et al. (2012), and (standard refers to BHVO-2, BIR-1, BCR-2 or USNM 3529 depending on the study considered). When the literature data were accompanied by two or more standards, the corrected $\mathrm{Tm} / \mathrm{Tm} *$ ratios were averaged. The corrections were small and similar when two or more standards were considered: about $3 \%$ for the data obtained by Dauphas and Pourmand (2015) and about $2 \%$ for the data obtained by Stracke et al. (2012). All values reported or discussed in this paper are relative to our BHVO-2 working values. 


\section{Results}

Results are given in Tables 1 to 4 . The REE abundances of the meteorites and terrestrial rocks analyzed here, are discussed extensively in the literature, and in depth discussions concerning the shape of the REE patterns will not be repeated here. Instead, our study is focused on Tm anomalies, which are small and not always discernable in the REE patterns, as demonstrated in Figure 3. The $\mathrm{Tm} / \mathrm{Tm} *$ ratios are presented in Figure 4 along with selected literature data. A synthesis of the $\mathrm{Tm} / \mathrm{Tm} *$ ratios in meteorites and bulk planetary bodies is given in Table 5 .

\subsection{Tm abundance in the CI reference}

Large chips of Orgueil and Ivuna were previously analyzed, and the average of five Orgueil samples was recommended for normalization purposes (Barrat et al., 2012). In order to check the normalization values for the heavy REEs, we dissolved $30 \mathrm{mg}$ splits of the previous CI powders (previous analyses of the same samples were obtained using about 120-150 mg of powders). The results display a limited range of $\mathrm{Tm} / \mathrm{Tm} *$ ratios from 0.991 to 1.003 (Table 2), with a mean $=0.999$ $(\sigma=0.005, \mathrm{n}=10)$. These results indicate that our CI average used for normalization does not require revision at the present level of precision. Pourmand et al. (2012) obtained Tm/Tm* ratios ranging from 0.999 to 1.043 , and consequently a CI average with a small positive anomaly relative to our results $(\mathrm{Tm} / \mathrm{Tm} *=1.022$ after correction of the interlaboratory bias). This small discrepancy could be at least

partially explained by the small size of most of the chips analyzed by these authors, totaling ca. $2.14 \mathrm{~g}$ for Orgueil, Alais and Ivuna, since it is well known that CI chondrites are heavily brecciated and chemically heterogeneous at the sub-mm scale (e.g., Morlok et al., 2006). It is also conceivable that CIs contain rare refractory inclusions (or their remnants after aqueous alteration) which could have affected their Tm abundances (Bendel et al., 2012a,b). Although one small CAI was found in Ivuna (Frank et al., 2011), the limited range of Tm/Tm* ratios shown by our samples is not consistent with the occurrence of heterogeneously dispersed refractory inclusions.

\subsection{Tm anomalies in other chondrites}

The abundance of $\mathrm{Tm}$ in chondrites has been measured in a large number of samples by Bendel et al. (2011, 2012a, b), Stracke et al. (2012), Khan et al. (2015), Pourmand et al. (2012) and Dauphas and Pourmand (2015). CM chondrites (Paris, Nogoya and Boriskino), enstatite chondrites (3 EH and 3 EL) and ordinary chondrites (Chelyabinsk and Braunschweig) have been analyzed here (Fig. 4 and Tables 2-3). Because all these chondrites have CE\#-numbers very close to 1 (=0.995-1.006, Table 2), their $\mathrm{Tm} / \mathrm{Tm}$ * ratios are unaffected by curvature in REE patterns and are faithful measures of $\mathrm{Tm}$ anomalies. The three $\mathrm{CM}$ samples display $\mathrm{Tm} / \mathrm{Tm} \mathrm{m}^{*}>1$ (i.e., from 1.009 to 1.036 ) contrary to the two ordinary (average $\mathrm{Tm} / \mathrm{Tm} *=0.972, \mathrm{n}=2$ ) and the six enstatite chondrites (average $\mathrm{Tm} / \mathrm{Tm} *=$ 
0.983, $\sigma=0.003, \mathrm{n}=6$ ) which all show small negative Tm anomalies. For all these types of chondrites, the new results are in good agreement with data obtained by Dauphas and Pourmand (2015). However, our results for enstatite chondrites appear much more homogeneous than the previously published data (Fig. 4 and Table 5).

\subsection{Tm anomalies in achondrites and pallasite}

Except for the Brenham olivine and NWA 7325, all our achondrite samples have CE\#-numbers very close to one $(=0.991-1.003$, Table 3$)$, indicating again that their $\mathrm{Tm} / \mathrm{Tm} *$ ratios are faithful measures of $\mathrm{Tm}$ anomalies. The $\mathrm{Tm} / \mathrm{Tm} *$ ratios measured on seven eucrites (four main group - Nuevo Laredo trend eucrites: Bereba, Juvinas, NWA 049 and Nuevo Laredo; three Stannern trend eucrites: Stannern, Bouvante, and NWA 2061) are very homogeneous, and range from 0.972 to 0.978 . Their average $\mathrm{Tm} / \mathrm{Tm} *$ ratio $(=0.974, \sigma=0.002)$ is similar to the average of ordinary chondrites $(=0.977, \sigma$ $=0.006, \mathrm{n}=22$, see Table 8 ).

Similar negative Tm anomalies were obtained for the angrite NWA 1296, and for five aubrites including the samples analyzed by Dauphas and Pourmand (2015). Moreover, the four ureilites analyzed here display analogous negative $\mathrm{Tm}$ anomalies $(\mathrm{Tm} / \mathrm{Tm} *=0.964-0.983)$, like the value measured previously for an Almahata Sitta trachyandesite $(\mathrm{Tm} / \mathrm{Tm} *=0.972)$ from the same parentbody (Bischoff et al., 2014). These results strengthen the "noncarbonaceous pedigree" of the ureilite parent body despite the high $\mathrm{C}$ contents of the ureilites, as previously suggested by Yamakawa et al. (2010) and Warren (2011a) from isotopic anomalies.

The REE pattern of the Brenham olivine is enriched in light REEs, with a slightly convex heavy REE distribution (see Fig. 7 in Greenwood et al., 2015). Indeed, the CE\#-number is low (=0.976) and the curvature of the pattern cannot be ignored. Although its $\mathrm{Tm} / \mathrm{Tm} *$ ratio $(=0.975)$ is similar to those of other achondrites, the $\mathrm{Tm} / \mathrm{Tm}{ }_{\mathrm{CE}}$ ratio is 0.999 indicating that no $\mathrm{Tm}$ anomaly is present in this pallasite. Other pallasites samples are necessary to determine the $\mathrm{Tm} / \mathrm{Tm} *$ ratio of the main-group and Eagle Station pallasite parent bodies.

NWA 7325 is an ungrouped achondrite, and it is the sole sample available on Earth from its parent body. It was suggested to have originated from Mercury (Irving et al., 2013), but it is too old to be a sample from the surface of this planet $(4562.5 \pm 4.4 \mathrm{Ma}$, Amelin et al., 2013; $4562.8 \pm 0.3 \mathrm{Ma}$, Dunlap et al., 2014). It formed from an unusual melt characterized by very low REE abundances and a very large positive Eu anomaly, generated by the remelting of an ancient gabbroic lithology. NWA 7325 is a remnant of one of the earliest crusts formed on a differentiated body recognized at present (Barrat et al., 2015). It displays the largest negative Tm anomaly analyzed so far in a magmatic rock (Fig. 3). Its 
$\mathrm{Tm} / \mathrm{Tm} *$ ratio $(=0.855)$ is too distinct to be explained solely by a curvature effect of the REE pattern despite a low CE\#-number $(=0.983)$. Indeed, its $\mathrm{Tm} / \mathrm{Tm}{ }_{\mathrm{CE}}$ ratio is similarly low $(=0.868)$. A possible explanation for this low value could be an analytical artifact. NWA 7325 displays a huge positive Eu anomaly, and consequently is characterized by a high Eu/Tm ratio of 313. In the case of Eu-rich rocks, the determination of $\mathrm{Tm}$ abundances could possibly be hampered by a ${ }^{153} \mathrm{Eu}^{16} \mathrm{O}$ interference on the

${ }^{169} \mathrm{Tm}$ peak, but the former is generally insignificant. This interference is monitored and corrected for in our procedure (e.g., Barrat et al., 1996). A negative Tm anomaly could be obtained if the Eu oxide interference was overcorrected. We have spiked a sample of BIR-1 with a solution of pure Eu, in order to check this effect. Although the Eu/Tm ratio of the spiked BIR-1 sample $(\mathrm{Eu} / \mathrm{Tm}=205$ instead of 2 in an unspiked sample) is very high, its $\mathrm{Tm} / \mathrm{Tm} *$ ratio is identical to the results obtained for unspiked samples (Table 1). We conclude that the low Tm/Tm* ratio obtained for NWA 7325 is real.

\subsection{Tm anomalies in Martian meteorites}

Six shergottites selected for this study range in composition from strongly light-REE depleted (Tissint and Dar al Gani 476) to enriched types (NWA 1669 and Los Angeles), including NWA 1950, one of the rare lherzolitic shergottites (Gillet et al., 2005). Their CE\# numbers range between 0.992 and 0.997, and indicate that the effects of the curvature of the patterns are insignificant. Indeed, the $\mathrm{Tm} / \mathrm{Tm} *$ ratios fall within a restricted range from 0.974 to 0.983 (average $\mathrm{Tm} / \mathrm{Tm} *=0.977, \sigma=$ $0.003)$, nearly identical to the eucrite and ordinary chondrite values.

\subsection{Tm anomalies in terrestrial and lunar rocks}

Three types of samples were selected: recent lavas ( $n=42$ including reference materials), mainly basalts, from all the oceans and from different geological settings (mid ocean ridge basalts, ocean island basalts, continental alkali basalts, island arc lavas), tektites (two indochinites and two Libyan Desert glasses) formed from the melting of sedimentary protoliths (e.g., Koeberl, 1992, Barrat et al., 1997), and a lunar meteorite.

Terrestrial lavas display a wide range of compositions, with a large diversity of REE patterns. Our sampling encompasses a large variety of lavas ranging from light-REE depleted oceanic basalts with no important curvature for the heavy REEs to alkali lavas with strongly fractionated patterns (e.g., lavas from Tubuai or from the French Massif Central). The curvature of the REE patterns is significant for some samples: the CE\#-numbers of the lavas range from 0.978 to 0.999. All lavas display a slight negative $\mathrm{Tm}$ anomaly and the $\mathrm{Tm} / \mathrm{Tm} *$ ratios show a significant range of variability, from 0.946 to 0.988 (Fig. 4). The mean $\mathrm{Tm} / \mathrm{Tm} *$ ratio $(=0.965, \sigma=0.011, \mathrm{n}=42)$ for all lavas including the reference materials BE-N, BIR-1, BHVO-2, JA3, and JB2, is slightly lower than the Vestan and Martian means. The spread of the $\mathrm{Tm} / \mathrm{Tm} *$ ratios is chiefly explained by a curvature effect of the REE 
patterns. Indeed, the samples with the lowest CE\#-numbers exhibit among the lowest $\mathrm{Tm} / \mathrm{Tm} *$ ratios (Fig. 5). A more limited range of values is obtained if $\mathrm{Tm} / \mathrm{Tm}^{*}{ }_{\mathrm{CE}}$ ratios are considered (Fig. 5).

Although the number of high quality Tm analyses of upper crustal rocks available at present is limited, their $\mathrm{Tm} / \mathrm{Tm} *$ ratios overlap and extend the range obtained for basaltic lavas to lower values (Fig. 4). The REE patterns of the two indochinites are nearly identical, with slight negative Tm anomalies (0.975-0.981) similar to those observed in shales (Figures 4 and 6). A Tm/Tm* as low as 0.933 was measured by Dauphas and Pourmand (2015) for G3 granite, a reference material. These authors interpreted the scatter in $\mathrm{Tm} / \mathrm{Tm}^{*}$ anomalies among terrestrial rocks to reflect the curvature of REE patterns and G3 is indeed a sample that displays a strong curvature (CE\# $=0.981$ ). The two Libyan Desert glasses show parallel REE patterns but with slightly more pronounced negative Tm anomalies than shales $(\mathrm{Tm} / \mathrm{Tm} *=0.947-0.954)$, and these may not totally explained by the curvature of the patterns (Fig. 6). Volatilization of Tm during impact is a possible explanation. However, Libyan Desert glasses display unfractionated isotope compositions of $\mathrm{Zn}$ and $\mathrm{Cu}$ in comparison to the terrestrial crust (Moynier et al., 2009, 2010). Because these elements are much more volatile than Tm, preferential volatilization of $\mathrm{Tm}$ during impact melting is unlikely. More high quality $\mathrm{Tm}$ measurements for upper crustal rocks (including granites and sediments), are necessary to evaluate a possible decoupling of Tm during geological processes.

Dhofar 460, a lunar feldspathic granulitic breccia paired with Dhofar 026, displays a small negative $\mathrm{Tm}$ anomaly $(\mathrm{Tm} / \mathrm{Tm} *=0.969)$, within error of the terrestrial lava mean.

\section{Discussion}

\subsection{Origin of the $\mathrm{Tm} / \mathrm{Tm} *$ anomalies in chondrites}

The dichotomy of Tm anomalies between carbonaceous and non-carbonaceous chondrites is confirmed by our new analyses. All carbonaceous chondrites analyzed so far display unresolved to positive $\mathrm{Tm}$ anomalies relative to $\mathrm{CI}$, while most of the non-carbonaceous chondrites exhibit negative $\mathrm{Tm}$ anomalies. These negative anomalies are subtle, on the order of just a couple of percent $(\mathrm{Tm} / \mathrm{Tm} *$ typically close to 0.975), and less marked than those calculated by Dauphas and Pourmand (2015) ( $\mathrm{Tm} / \mathrm{Tm} *$ typically close to 0.96 ), due to the different $\mathrm{CI}$ averages used for normalization.

Large positive Tm anomalies in some carbonaceous chondrites are well known since a long time, notably in Allende for which they have been interpreted as fingerprints of the presence of group II CAIs (Fig. 6, e.g., Mason and Taylor, 1982; Shinotsuka et al., 1995; Stracke et al., 2012). Thus, it can be inferred that the range of $\mathrm{Tm} / \mathrm{Tm} *$ ratios in chondrites is mostly controlled by the distribution 
of refractory inclusions or other refractory components. Indeed, Dauphas and Pourmand (2015) pointed out that there is a relationship between Tm anomalies and $\mathrm{Ca}$ isotopic fractionation in bulk meteorites. The reason is that CAIs with type II REE patterns also have fractionated Ca isotopic compositions (Huang et al., 2012). Ordinary and enstatite chondrites have slight negative Tm anomalies and $\delta^{44} \mathrm{Ca}$ values close to the terrestrial composition, while carbonaceous chondrites have positive $\mathrm{Tm}$ anomalies and heavy $\delta^{44} \mathrm{Ca}$ values relative to Earth. The data plot on mixing trends defined by type II CAIs and by the terrestrial composition as endmembers (Fig. 7). This general pattern is confirmed by the present study but in order to make progress on this topic, one would need to measure $\mathrm{Ca}$ isotopes and $\mathrm{Tm}$ anomalies in the same aliquots, as carbonaceous chondrites are likely to be heterogeneous at the sampling scale. Some of the carbonaceous chondrites with high $\mathrm{Tm} / \mathrm{Tm} *$ values relative to inner solar system bodies are devoid of CAIs (e.g., CIs). The carriers of Tm anomalies need not be in the form of grains that aggregated to form CAIs, however. They could also be in the form of disseminated refractory dust grains that carry the same chemical signature but were obliterated by parent-body processes such as aqueous alteration on the CI parent-body. This means that a simple correlation between the modal abundance of CAIs and Tm anomalies is neither expected nor observed.

\subsection{Tm/Tm* ratios of the bulk achondrite parent bodies, Mars and Earth}

Because melting and fractional crystallization most likely do not fractionate Tm abundances from those of $\mathrm{Er}$ and $\mathrm{Yb}$, the $\mathrm{Tm} / \mathrm{Tm} *$ ratios of magmatic rocks or mantle restites from a given planetary body provide a picture of the heterogeneity of the body for this parameter. The restricted ranges of $\mathrm{Tm} / \mathrm{Tm} *$ ratios exhibited by aubrites, ureilites, eucrites and shergottites indicate that their parent bodies accreted from homogeneous building blocks, or were efficiently homogenized by differentiation processes. Consequently, their mean $\mathrm{Tm} / \mathrm{Tm} *$ ratios are certainly very good estimates of the $\mathrm{Tm} / \mathrm{Tm} *$ of their parent bodies (Table 5 ).

The determination of the Tm anomaly of the bulk Earth is not as straightforward as for those of other telluric bodies, because terrestrial rocks, including lavas and continental rocks, display a puzzling range of $\mathrm{Tm} / \mathrm{Tm} *$ ratios, from 0.93 to 0.99 , which is chiefly explained by the diversity and curvature of their REE patterns (also see Dauphas and Pourmand, 2015). The mean Tm/Tm* ratios of all lavas cannot provide a correct estimate for the bulk Earth because this mean is certainly biased by the alkali lavas, which display the lowest CE\#-numbers and $\mathrm{Tm} / \mathrm{Tm} *$ ratios, and comprise about half of our database. Instead, we estimate the $\mathrm{Tm} / \mathrm{Tm} *$ ratio of the bulk Earth using only lava samples for which the curvature effect is negligible, in other words those samples with CE\# numbers in the range $0.99-1.00$. Thus, the $\mathrm{Tm} / \mathrm{Tm} *$ ratio of our planet is certainly very close to 0.976 (Table 5). This ratio is similar to the mean of the shales $(\mathrm{Tm} / \mathrm{Tm} *=0.974$, Table 5) whose CE\# numbers are all in the range 
0.996-1.003 (Fig. 5). The mean of the shales certainly mirrors the upper continental crust value, and suggests that Tm was not decoupled from other heavy REEs during the formation of the continental crust or sedimentary processes. The lower $\mathrm{Tm} / \mathrm{Tm} *$ ratio $(=0.963, \mathrm{n}=25)$ displayed by the mean of the aeolian dust analyzed by Pourmand et al. (2014) is not explained at present (Fig. 5), but a slight analytical bias cannot be excluded (Fig. 5).

\subsection{Tm/Tm* ratios and non-CI refractory lithophile element abundances}

An important observation that emerges from our work is that the $\mathrm{Tm} / \mathrm{Tm} *$ ratios of the Earth, Mars, Vesta, the UPB, and the aubrite parent body are indistinguishable and close to 0.975 (Fig. 4 and Table 5). This may be a characteristic signature of inner solar system solids (Dauphas and Pourmand, 2015). This inference is strengthened by the results obtained on one angrite, and on ordinary and enstatite chondrites, which all display similar $\mathrm{Tm} / \mathrm{Tm} *$ ratios as Earth, Mars, and Vesta. The unique achondrite NWA 7325, which crystallized from an impact melt, is the sole sample with a much lower $\mathrm{Tm} / \mathrm{Tm} *$ ratio. Its parent body could have formed from a minor reservoir with distinctly lower negative Tm anomalies.

The homogeneity of the Tm/Tm* ratios in the bulk Earth, Mars and other small differentiated bodies has important implications. $\mathrm{Tm} / \mathrm{Tm} *$ ratios are very sensitive to the proportions of group II CAIs in primary materials. Using the mean of the group II CAIs analyzed by Huang et al. (2012) and CI abundances, calculations indicate that $\mathrm{Tm} / \mathrm{Tm} *$ ratios ranging from 0.97 to 0.98 can be explained by variations in the proportion of Group II CAI-like dust in the order of $\pm 0.035 \mathrm{wt} \%$ only. Thus the materials that formed the Earth and Mars, and much smaller differentiated bodies like Vesta or the aubrite parent body, were extremely homogeneous with respect to heavy REE fractionation, despite a strong heterogeneity in terms of volatile depletions (Fig. 8).

Refractory lithophile elements (RLEs; Ca, Al, Ti, REEs) are usually assumed to be in CI-like proportions in planetary materials, reflecting the fact that they cannot easily be fractionated at a bulk planetary scale by nebular processes. This assumption is very difficult to put to the test because these elements can be fractionated by magmatic and aqueous processes that overprint earlier signatures. The first line of evidence that the Earth may have non-chondritic RLE ratios came from high precision

${ }^{142} \mathrm{Nd}$ isotope measurements which revealed that the accessible Earth may have a Sm/Nd ratio higher than chondritic (Boyet and Carlson, 2005). Nebular processes cannot readily explained this fractionation and it was suggested that either a hidden early formed enriched reservoir with a low $\mathrm{Sm} / \mathrm{Nd}$ is present in the deep Earth (i.e., a recycled protocrust; Boyet and Carlson, 2005) or a protocrust with low $\mathrm{Sm} / \mathrm{Nd}$ was lost to space by impact erosion (Caro et al. 2008). Either way, the Earth would have started with a chondritic $\mathrm{Sm} / \mathrm{Nd}$ ratio. Interpretation of ${ }^{142} \mathrm{Nd}$ is still a matter of contention as subsequent measurements showed that ${ }^{142} \mathrm{Nd}$ variations could be partly explained by the 
presence of nucleosynthetic anomalies (Gannoun et al., 2011), so that the silicate Earth may still have chondritic $\mathrm{Sm} / \mathrm{Nd}$ ratio. The finding of $\mathrm{Tm}$ anomalies in bulk planetary materials relative to CIs (this study; Dauphas and Pourmand 2015) demonstrates that the assumption that RLEs are present in CI proportions in Earth and other terrestrial planets is not valid. CIs are enriched in a highly refractory dust component with fractionated RLE abundances relative to inner solar system objects.

Finally, the contribution of carbonaceous chondrites during the main stages of the accretion of the terrestrial planets has been a matter of debates. Many models have been put forward ranging from the involvement of essentially non-carbonaceous chondrites for the Earth and Mars to various proportions of carbonaceous and non-carbonaceous chondrites for the Earth, Mars and Vesta (Sanloup et al., 1999; Lodders, 2000; Burbine and O'Brien, 2004; Warren, 2011b; Javoy et al., 2010; Toplis et al., 2013; Siebert et al., 2013; Dauphas et al., 2014 and references therein). In principle, the Tm anomalies in extraterrestrial materials could offer new constraints for estimates of the proportions of carbonaceous chondrites in terrestrial bodies. Unfortunately the $\mathrm{Tm} / \mathrm{Tm} *$ ratio is not very sensitive to the contributions of such material: for example a $75 \%$ ordinary chondrite $+25 \% \mathrm{CM}$ mixture [calculated with Chelyabinsk $(\mathrm{Tm} / \mathrm{Tm} *=0.975)$ and Paris $(\mathrm{Tm} / \mathrm{Tm} *=1.009)]$ displays a $\mathrm{Tm} / \mathrm{Tm} *=0.983$ just slightly higher than the mean ordinary chondrite value, at the limit of the present level of precision. However, the homogeneity of the $\mathrm{Tm} / \mathrm{Tm} *$ ratios for the Earth, Mars and Vesta strongly suggests that carbonaceous chondrites were not the main planetary building materials in the Inner Solar System, in agreement with the systematics of $\mathrm{O}, \mathrm{Ca}, \mathrm{Ti}, \mathrm{Cr}$, and Ni isotopes (e.g., Warren, 2011b; Dauphas et al., 2014).

\section{Conclusions}

Our analyses of chondrites confirm the dichotomy between carbonaceous and non-carbonaceous chondrites reported by previous studies. Relative to CIs, carbonaceous chondrites display $\mathrm{Tm} / \mathrm{Tm} *$ ratios equal to, or higher than one, while the vast majority of the non-carbonaceous chondrites display negative $\mathrm{Tm} / \mathrm{Tm} *$ anomalies (Fig. 4). All achondrites and planetary samples analyzed so far display small negative $\mathrm{Tm}$ anomalies relative to CIs $(\mathrm{Tm} / \mathrm{Tm} *=0.975)$, similar to non-carbonaceous chondrites (Fig. 4). Terrestrial rocks show variable $\mathrm{Tm} / \mathrm{Tm} *$ ratios that reflect the fact that the use of $\mathrm{Er}$ and $\mathrm{Yb}$ abundances for normalization to determine Tm anomalies can lead to misleading results if REE patterns display some curvature. Accounting for this curvature effect, we show that the Earth has a $\mathrm{Tm} / \mathrm{Tm} *$ ratio of 0.976 . One of the most important observations that emerges from our work is that the mean $\mathrm{Tm} / \mathrm{Tm}^{*}$ ratios of the Earth, Mars, 4-Vesta, and possibly of most of the parent bodies of achondrites (angrites, ureilites, aubrites) are similar. This suggests that small negative Tm anomalies (typically $\mathrm{Tm} / \mathrm{Tm} *=0.975$ ) were a widespread feature of inner solar system solids. Our data agree with isotope studies, which suggest that carbonaceous chondrites represent a minor fraction of Earth's 
building blocks. The presence of negative Tm anomalies in bulk planetary materials (Earth, Mars, Vesta, other achondrite parent bodies) relative to CIs demonstrates that the assumption that refractory lithophile elements are present in CI proportions in planets is unwarranted as CIs are seemingly enriched in a refractory component with a distinct RLE signature.

\section{Acknowledgements}

The samples analyzed during the course of this study were kindly provided to the first author during the last 25 years by the Muséum National d'Histoire Naturelle (Paris), Ifremer, the Université Pierre et Marie Curie, the Université de Rennes 1, the Institute for Meteoritics, Albuquerque, the National Institut of Polar Research, Tokyo, the Natural History Museum, Vienna, the Smithsonian Institution, the Meteorite Working Group (NASA), Patrick Bachélery, Ted Bunch, Alain et Louis Carion, Martial Caroff, Bruno and Carine Fectay, Luc Labenne, Robert Roccia, Don Simptson, and our late colleagues Theodore Monod and Jean-Louis Cheminée. US Antarctic meteorite samples are recovered by the Antarctic search for Meteorites (ANSMET) program which has been funded by NSF and NASA, and characterized and curated in the department of Mineral Sciences of the Smithsonian Institution and Astromaterials Curation Office at NASA Johnson Space Center. Special thanks to Frederik Paulsen who kindly provided a large sample of Chelyabinsk. We thank Marc Norman and Mark Rehkämper for the editorial handling and their helpful comments which greatly improved the manuscript, Christine Floss, Herbert Palme, two anonymous reviewers for their constructive comments and Pascale Barrat for her help. This work was supported by the "Laboratoire d'Excellence" LabexMER (ANR-10-LABX-19) and co-funded by grants from the French government under the program "Investissements d'Avenir" and from the Programme National de Planétologie (CNRS-INSU) to the first author. 


\section{References}

Amelin Y., Koefoed P., Iizuka T., and Irving A.J. (2013) U-Pb age of ungrouped achondrite NWA 7325. 76 ${ }^{\text {th }}$ Annual Meteoritical Society Meeting, abstract \#5165.

Anders E. and Grevesse N. (1989) Abundances of the elements: meteoritic and solar. Geochim. Cosmochim. Acta 53, 197-214.

Barrat J.A., Keller F., Amossé J., Taylor R.N., Nesbitt R.W., Hirata T. (1996) Determination of rare earth elements in sixteen silicate reference samples by ICP-MS after Tm addition and ion exchange separation. Geostandards Newsletter 20, 1, 133-140.

Barrat J.A., Jahn B.M., Amossé J., Rocchia R., Keller F., Poupeau G.R., Diemer E. (1997). Geochemistry and origin of Libyan Desert glasses. Geochim. Cosmochim. Acta 61, 1953-1959.

Barrat J.A., Zanda B., Moynier F., Bollinger C., Liorzou C., and Bayon G. (2012) Geochemistry of CI chondrites: Major and trace elements, and $\mathrm{Cu}$ and $\mathrm{Zn}$ isotopes. Geochim. Cosmochim. Acta 83, 79-92.

Barrat J.A., Zanda B., Jambon A., Bollinger C. (2014) The lithophile trace elements in enstatite chondrites. Geochim. Cosmochim. Acta. 128, 71-94.

Barrat J.A., Greenwood R.C., Verchovsky A.B., Gillet Ph., Bollinger C., Liorzou C., Franchi I.A. (2015) Crustal differentiation in the early solar system: clues from the unique achondrite Northwest Africa 7325 (NWA 7325). Geochim. Cosmochim. Acta. 168, 280-292.

Bayon G., Barrat J.A., Etoubleau J., Benoit M., Révillon S. (2009) Determination of Sc, Y, Zr, Ba, Hf, Th and REE in geological samples by ICP-MS after Tm addition and alkaline fusion. Geostandards and Geoanalytical Research 33, 51-62.

Bendel V., Patzer A., Pack A., Hezel D.C., Münker C. (2011) Rare Earth Elements in bulk chondrites and chondrites components. $42^{\text {nd }}$ Lunar and Planetary Science Conference, abstract \# 1711.

Bendel V., Pack A., O’Neill H. St. C. (2012a) Rare Earth Elements in CI1-chondrites and planetary samples. $43^{\text {rd }}$ Lunar and Planetary Science Conference, abstract \# 2578.

Bendel V., Pack A., O’Neill H. St. C., Jenner F.E., Münker C. (2012b) Volatility-controlled rare earth element fractionation in the early solar system. $75^{\text {th }}$ Annual Meteoritical Society Meeting, abstract \# 5351.

Bischoff A., Horstmann M., Barrat J.A., Chaussidon M., Pack A., Herwartz D., Ward D., Vollmer C., Decker S. (2014) Trachyandesitic volcanism in the early Solar System - constraints from the ureilite parent body. PNAS 111, 35, 12689-12692.

Boyet M. and Carlson R. W. (2005) ${ }^{142} \mathrm{Nd}$ evidence for early (>4.53 Ga) global differentiation of the silicate Earth. Science 214, 427-442.

Boynton W. V. (1975) Fractionation in the solar nebula: condensation of yttrium and the rare earth elements. Geochim. Cosmochim. Acta 39, 569-584.

Burbine T.H., O'Brien K.M. (2004) Determining the possible building blocks of the Earth and Mars. Meteoritics and Planetary Science 39, 667-681.

Caro G., Bourdon B., Halliday A. N. and Quitté G. (2008) Superchondritic Sm/Nd ratios in Mars, the Earth and the Moon. Nature 452, 336-339.

Coryell C.D., Chase J. W., and Winchester J.W. (1963) A procedure for geochemical interpretation of terrestrial rare-earth abundance patterns. J. Geophys. Res. 68, 559-566.

Dauphas N., Chen J.H., Zhang J., Papanastassiou D.A., Davis A.M., Travaglio C. (2014) Calcium-48 isotopic anomalies in bulk chondrites and achondrites: evidence for a uniform isotopic reservoir in the inner protoplanetary disk. Earth and Planetary Science Letters 407, 96-108. 
Dauphas N, Pourmand A. (2015) Thulium anomalies and rare earth element patterns in meteorites and Earth: Nebular fractionation and the nugget effect. Geochim. Cosmochim. Acta 163, 234-261.

Davis A. M., Grossman L. (1979) Condensation and fractionation of rare earths in the solar nebula. Geochim. Cosmochim. Acta 43, 1611-1632.

Dunlap D. R., Wadhwa M., Romaneillo S. R. (2014) ${ }^{26} \mathrm{Al}-{ }^{26} \mathrm{Mg}$ systematics in the unusual ungrouped achondrite NWA 7325 and the eucrite Juvinas. $\mathbf{4 5}^{\text {th }}$ Lunar Planet. Sci. Conf., abstract \# 2186.

Fegley B. and Ireland T. R. (1991) Chemistry of the rare earth elements in the solar nebula. Eur. J. Solid State Inorg. Chem. 28, 335-346.

Frank D., Zolensky M., Martinez J., Mikouchi T., Ohsumi K., Hagiya K., Satake W., Le L., Ross D., Peslier A. (2011) A CAI in the Ivuna CI1 chondrite. $42^{\text {nd }}$ Lunar and Planetary Science Conference, abstract \#2785.

Frey F.A., Green D.H., Roy S.D. (1978) Integrated models of basalt petrogenesis - Study of quartz tholeiites to olivine melilitites from South Eastern Australia utilizing geochemical and experimental petrological data. $J$. Petrology 19, 463-513.

Gannoun A., Boyet M., Rizo H., El Goresy A. (2011) ${ }^{146} \mathrm{Sm}-{ }^{142} \mathrm{Nd}$ systematics measured in enstatite chondrites reveals a heterogeneous distribution of ${ }^{142} \mathrm{Nd}$ in the solar nebula. PNAS 108, 19, 7693-7697.

Gillet Ph., Barrat J.A., Beck P., Marty B., Greenwood R.C., Franchi I.A., Bohn M., Cotten J. (2005) Petrology, geochemistry, and cosmic-ray exposure age of Northwest Africa 1950. Meteoritics Planet. Sci. 40, 1175-1184.

Greenwood R.C., Barrat J.A., Scott E.R.D., Haack H., Buchanan P.C., Franchi I.A., Yamaguchi A., Johnson D., Bevan A.W.R., Burbine T.H. (2015) Geochemistry and oxygen isotope composition of main-group pallasites and olivine-rich clasts in mesosiderites: Implications for the "Great Dunite Shortage" and HED-mesosiderite connection. Geochim. Cosmochim. Acta 169, 115-136.

Hanson G.N. (1980) Rare Earth Elements in petrogenetic studies of igneous systems. Ann. Rev. Earth Planet. Sci. 8, 371-406.

Hewins R.H., Bourot-Denise M., Zanda B., Leroux H., Barrat J.A., Humayun M., Göpel C., Greenwood R.C., Franchi I.A., Pont S., Lorand J.P., Cornède C., Gattacceca J., Rochette P., Kuga M., Marrocchi Y., Marty B. (2014) The Paris meteorite, the least altered CM chondrite. Geochim. Cosmochim. Acta. 124, 190-222.

Hiyagon H., Yamakawa A., Ushikubo T., Lin Y., Kimura M. (2011) Fractionation of rare earth elements in refractory inclusions from the Ningqiang meteorite: origin of positive anomalies in $\mathrm{Ce}, \mathrm{Eu}$ and $\mathrm{Yb}$. Geochim. Cosmochim. Acta 75, 3358-3384.

Huang S., Farkas J., Yu G., Petaev M.I., Jacobsen S.B. (2012) Calcium isotopic ratios and rare earth element abundances in refractory inclusions from Allende CV3 chondrite. Geochim. Cosmochim. Acta 77, $252-265$.

Irving A.J., Kuehner S.M., Bunch T.E., Ziegler K., Chen G., Herd C.D.K., Conrey R.M., Ralew S. (2013) Ungrouped mafic achondrite Northwest Africa 7325: a reduced iron-poor cumulate olivine gabbro from a differentiated planetary parent body. $44^{\text {th }}$ Lunar and Planetary Science Conference, abstract \# 2164.

Javoy M., Kaminski E., Guyot F., Andrault D., Sanloup C., Moreira M., Labrosse S., Jambon A., Agrinier P., Davaille A., Jaupart C. (2010) The chemical composition of the Earth: enstatite chondrite models. Earth Planet. Sci. Lett. 293, 259-268.

Khan R., Shirai N., Ebihara M. (2015) Chemical characteristic of R chondrites in the light of P, REEs, Th and U abundances. Earth Planet. Sci. Lett. 422, 18-27.

Koeberl C. (1992) Geochemistry and origin of Muong Nong -type tektites. Geochim. Cosmochim. Acta 56, 1033-1064. 
Lodders K. (2000) an oxygen isotope mixing model for the accretion and composition of rocky planets. Space Science Reviews 92, 341-354,

Lodders K. (2003) Solar system abundances and condensation temperatures of the elements. Astrophys. J. 591, $1220-1247$.

Magna T., Gussone N., Mezger K. (2015) The calcium isotope systematics of Mars. Earth Planet. Sci. Lett. 430, 86-94.

Masuda A. (1962) Regularities in variation of relative abundances of Lanthanide elements and an attempt to analyse separation index patterns of some minerals. J. Earth Sci. Nagoya Univ. 10, 173-187.

MacPherson G.J. (2003) Calcium-aluminum-rich inclusions in chondritic meteorites. In meteorites, Comets and Planets (ed. A. M. Davis) Vol. 1 Treatise on Geochemistry (eds. H.D. Holland and K.K. Turekian), pp. 201-246 Elsevier-Pergamon, Oxford.

Mason B., Taylor S.R. (1982) Inclusions in the Allende meteorite. Smithsonian Contrib. Earth Sci. 25, pp 1-30.

Moynier F., Beck P., Jourdan F., Yin Q.-Z., Reimold W. U. and Koeberl C. (2009) Isotopic fractionation of zinc in tektites. Earth Planet. Sci. Lett. 277, 482-489.

Moynier F., Koeberl C., Beck P., Jourdan F., Telouk P. (2010) Isotopic fractionation of $\mathrm{Cu}$ in tektites. Geochimica Cosmochimica Acta 74, 799-807.

Morlok A., Bischoff A., Stephan T., Floss C., Zinner E., Jessberger E.K. (2006) Brecciation and chemical heterogeneities of CI chondrites. Geochimica et Cosmochimica Acta 70, 5371-5394.

Nance W.B., Taylor S.R., (1976) Rare earth element patterns and crustal evolution-I. Australian post-Archean sedimentary rocks. Geochimica et Cosmochimica Acta 40, 1539-1551.

Pack A., Shelley J. M. G. and Palme H. (2004) Chondrules with peculiar REE patterns: implications for nebular condensation at high C/O. Science 303, 997-1000.

Palme H., Lodders K. and Jones A. (2014) Solar System Abundances of the Elements. In: Holland H.D. and Turekian K.K. (eds.) Treatise on Geochemistry, Second Edition, vol. 2, pp. 15-36. Oxford: Elsevier.

Pourmand, A., Dauphas, N., Ireland, T.J. (2012) A novel extraction chromatography and MC-ICP-MS technique for rapid analysis of REE, Sc and Y: Revising CI-chondrite and Post-Archean Australian Shale (PAAS) abundances. Chem. Geol. 291, 38-54.

Pourmand A., Prospero J., Sharifi M., Arash. (2014) Geochemical fingerprinting of trans-Atlantic African dust based on radiogenic Sr-Nd-Hf isotopes and rare earth element anomalies. Geology, doi:10.1130/g35624.1

Raczek I., Stoll B., Hofmann A. W. and Jochum K. P. (2001) High precision trace element data for the USGS reference materials BCR-1, BCR-2, BHVO-1, BHVO-2, AGV-1, AGV-2, DTS-1,DTS-2, GSP-1 and GSP-2 by ID-TIMS and MIC SSMS. Geostand. Newsl. 25, 77-86.

Sanloup C., Jambon A., Gillet P. (1999) A simple chondritic model of Mars. Phys. Earth Planet. Interiors 112, 43-54.

Shinotsuka K., Hidaka H. and Ebihara M. (1995) Detailed abundances of rare earth elements, thorium and uranium in chondritic meteorites: an ICP-MS study. Meteoritics 30, 694-699.

Siebert J., Badro J., Antonangeli D., Ryerson F.J. (2013) Terrestrial accretion under oxidizing conditions. Science 339, 1194-1197.

Stracke A., Palme H., Gellissen M., Münker C., Kleine T., Birbaum K., Detlef G., Bourdon B., Zipfel J. (2012) Refractory element fractionation in the Allende meteorite: Implications for solar nebula condensation and the chondritic composition of planetary bodies. Geochim. Cosmochim. Acta 85, 114-141. 
Tanaka T., Masuda A. (1973) Rare Earth Elements in matrix, inclusions, and chondrules of the Allende meteorite. Icarus 19, 523-530.

Taylor S.R., McLennan S.M. (1981) The composition and evolution of the continental crust: rare earth element evidence from sedimentary rocks. Phil. Trans Royal Soc. London Ser. A, 301, 381-399.

Toplis M.J., Mizzon H., Monnereau M., Forni O., McSween H.Y., Mittlefehldt D.W., McCoy T.J., Prettyman T.H., De Sanctis M.C., Raymond C.A., Russell C.T. (2013) Chondritic models of 4 Vesta; implications for geochemical and geophysical properties. Meteoritics Planetary Sci. 48, 2300-2315.

Valdes M.C., Moreira M., Foriel J., Moynier F. (2014) The nature of Earth's building blocks as revealed by calcium isotopes. Earth Planet. Sci. Let., 394, 135-145.

Warren P.H. (2011a) Stable isotopes and the noncarbonaceous derivation of ureilites, in common with nearly all differentiated planetary materials. Geochim. Cosmochim. Acta 75, 6912-6926.

Warren P.H. (2011b) Stable-isotopic anomalies and the accretionary assemblage of the Earth and Mars: A subordinate role for carbonaceous chondrites. Earth Planet. Sci. Lett., 311, 93-100.

Yamakawa A., Yamashita K., Makashima A. and Nakamura E. (2010) Chromium isotope systematics of achondrites: chronology and isotopic heterogeneity of the inner solar system. Astrophys. J. 720, 150-154. 


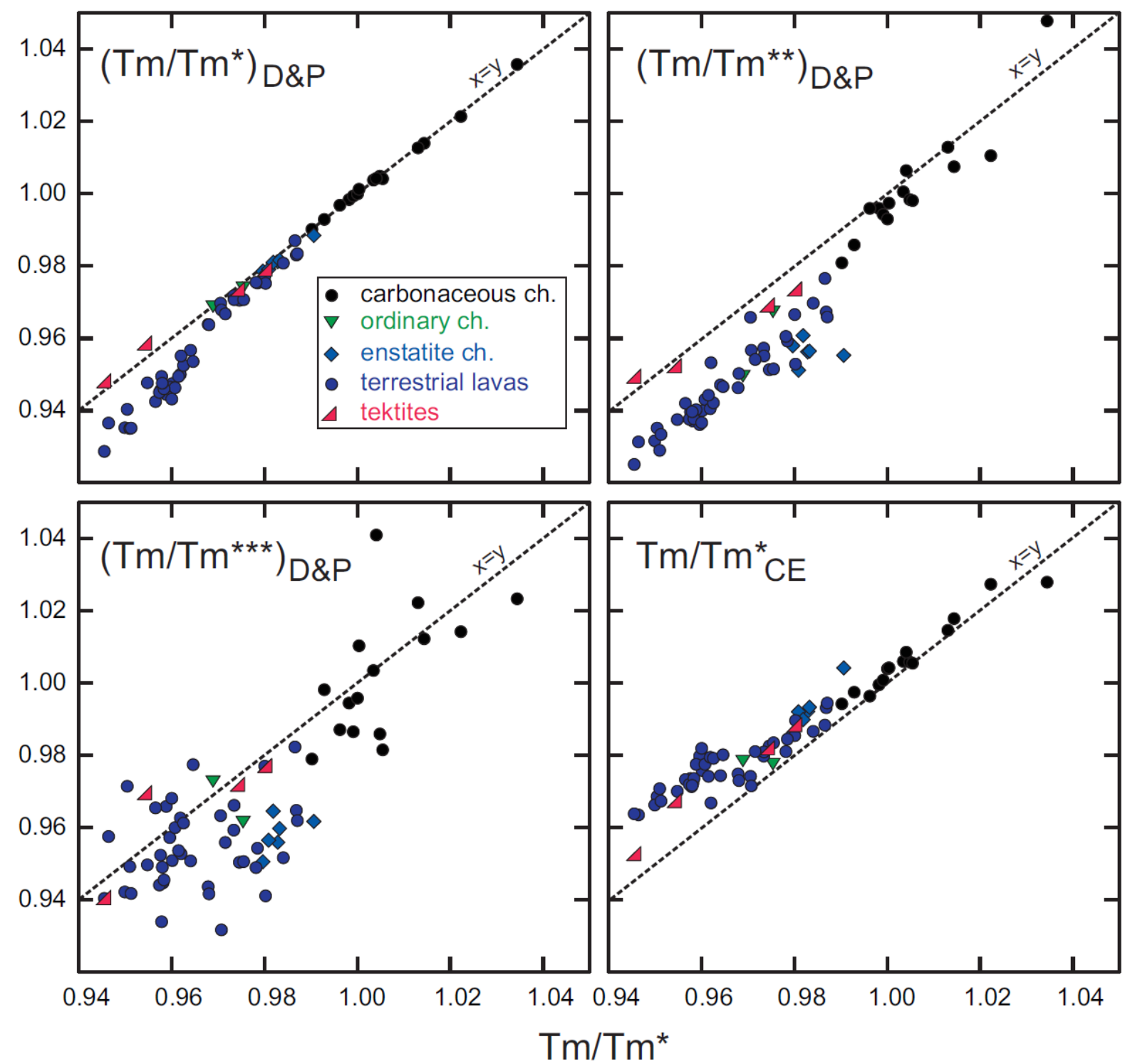

Fig. 1. Comparison of the $\mathrm{Tm} / \mathrm{Tm} *, \mathrm{Tm} / \mathrm{Tm} * *, \mathrm{Tm} / \mathrm{Tm} * * *$ ratios calculated using the equations given by Dauphas and Pourmand (2015), and $\mathrm{Tm} / \mathrm{Tm}{ }^{*}{ }_{\mathrm{CE}}$ with the $\mathrm{Tm} / \mathrm{Tm} *$ ratios proposed in this study, for a selection of chondrites and terrestrial rocks. 

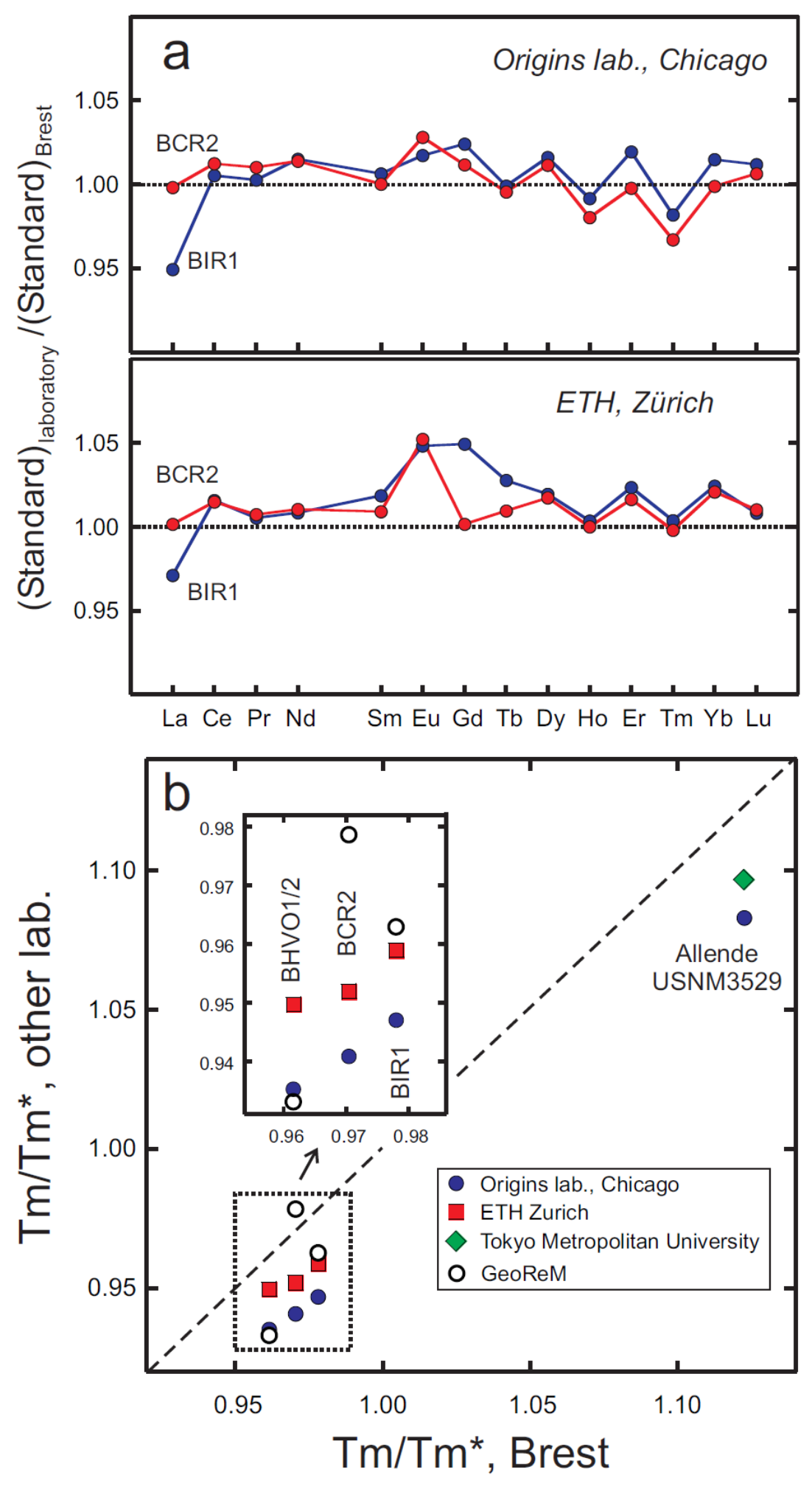

Fig. 2. Comparison of the REE abundances (a), and the $\mathrm{Tm} / \mathrm{Tm} *$ ratios for reference materials obtained during the course of this study in Brest, with those, uncorrected, obtained at ETH (Stracke et al., 2012), Origins laboratory (Pourmand et al., 2012), Tokyo Metropolitan University (Khan et al., 2015) and the GEOREM's preferred values (georem.mpch-mainz.gwdg.de). Tm/Tm* ratios are relative to the CI data recommended by Barrat et al. (2012). Although the Tm/Tm* values obtained in the different laboratories vary slightly, the results are highly coherent. 


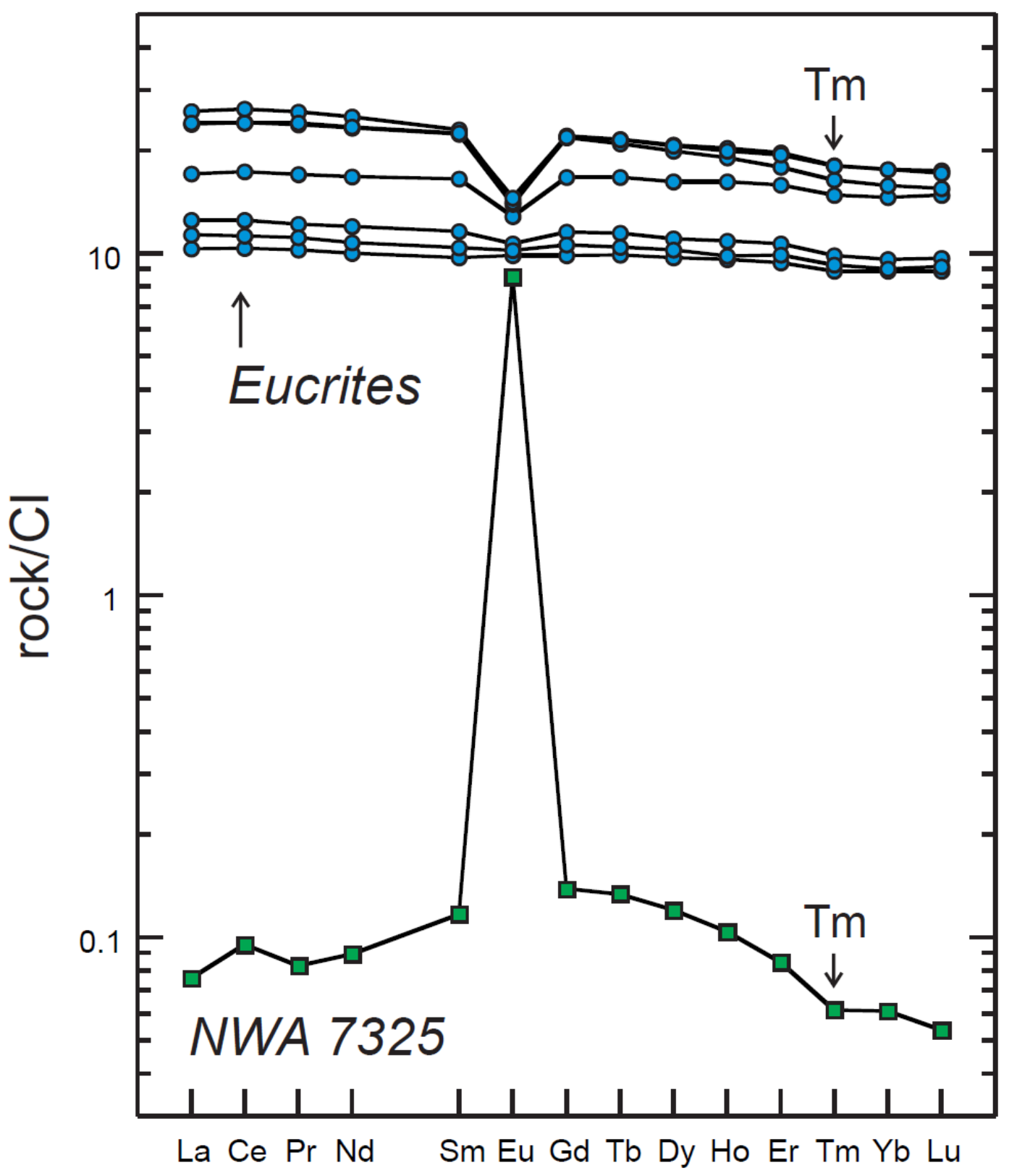

Fig. 3. REE patterns for eucrites and the ungrouped achondrite NWA 7325 normalized to CI chondrite (Barrat et al., 2012). The negative Tm anomalies are generally too small to be easily discerned on the REE patterns of achondrites or planetary samples, excepted for NWA 7325. 


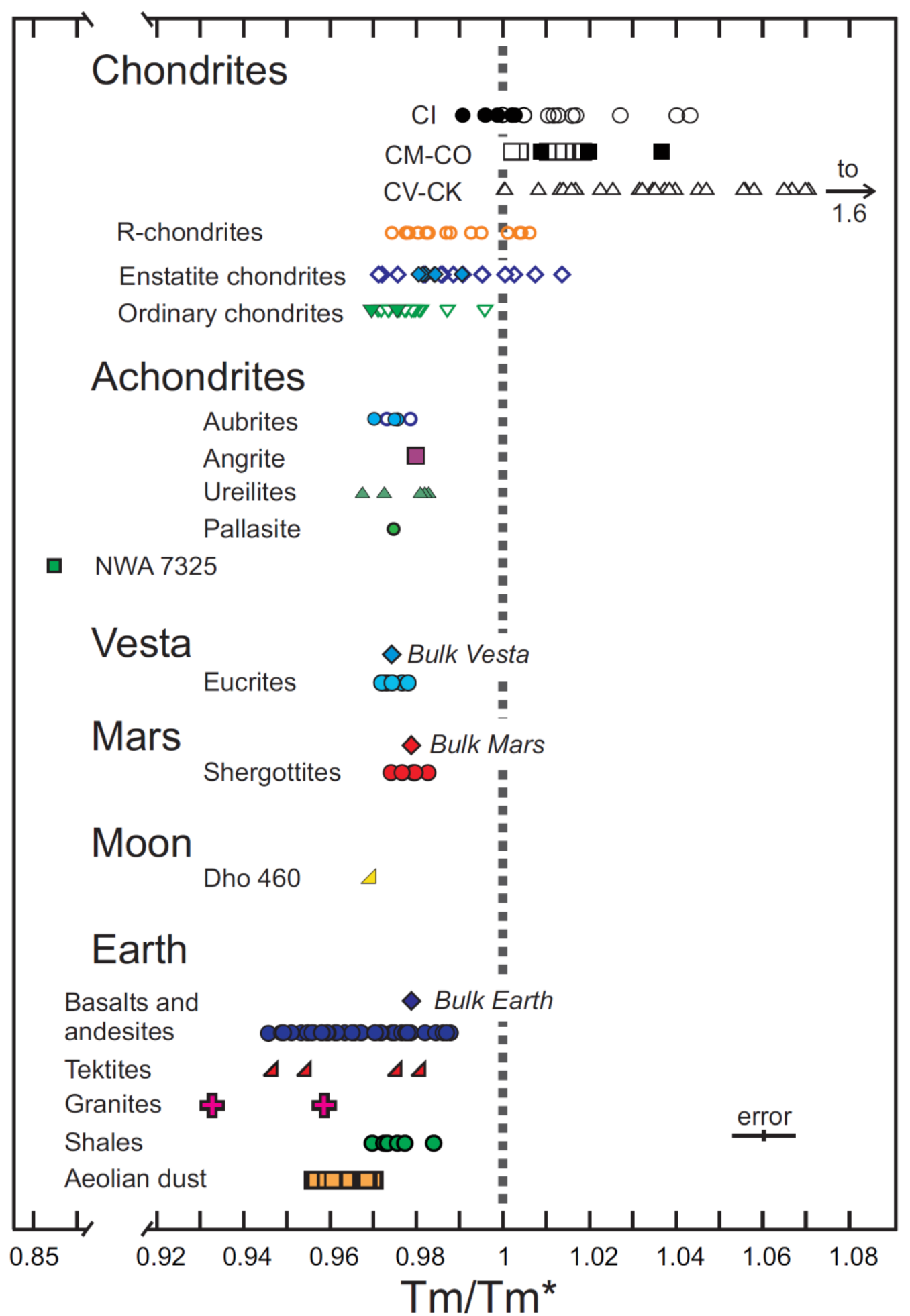

Fig. 4. Tm/Tm* ratios relative to $\mathrm{CI}$ among meteorites and planetary samples (open symbols: Pourmand et al., 2012; Stracke et al., 2012; Bischoff et al., 2012; Dauphas and Pourmand, 2015; Khan et al., 2015; filled symbols: this study). Literature data are adjusted to the Brest BIR-1 and BCR-2 values and $\mathrm{Tm} / \mathrm{Tm} *$ ratios are relative to the $\mathrm{CI}$ data recommended by Barrat et al. (2012). 

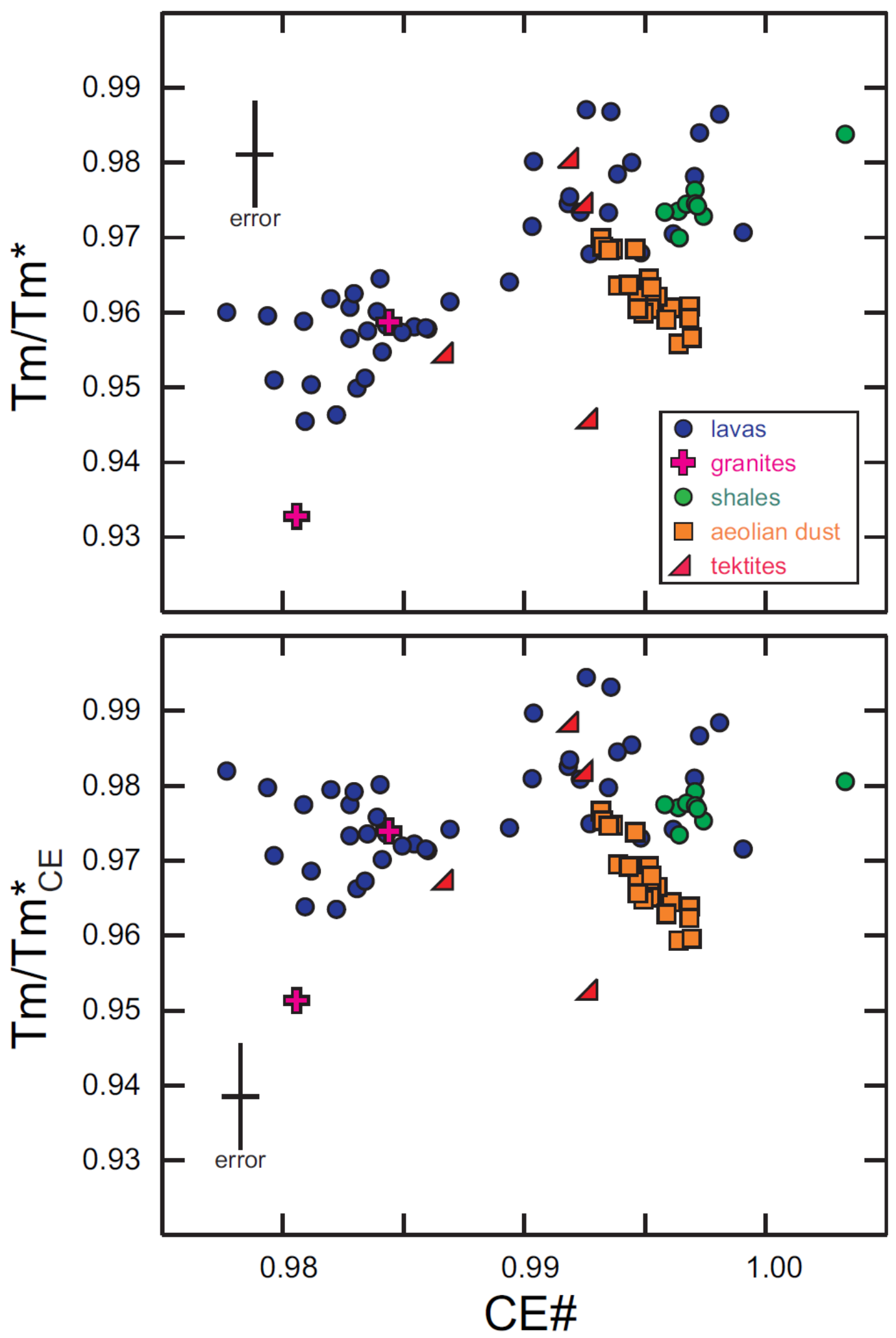

Fig. 5. Tm/Tm* and $\mathrm{Tm} / \mathrm{Tm}{ }^{\mathrm{CE}}{ }_{\mathrm{CE}}$ vs. CE\# plots for terrestrial samples (Pourmand et al., 2012, 2014; this study). Literature data are adjusted to the Brest BIR-1 and BCR-2 values and $\mathrm{Tm} / \mathrm{Tm} *$ ratios are relative to the CI data recommended by Barrat et al. (2012). 


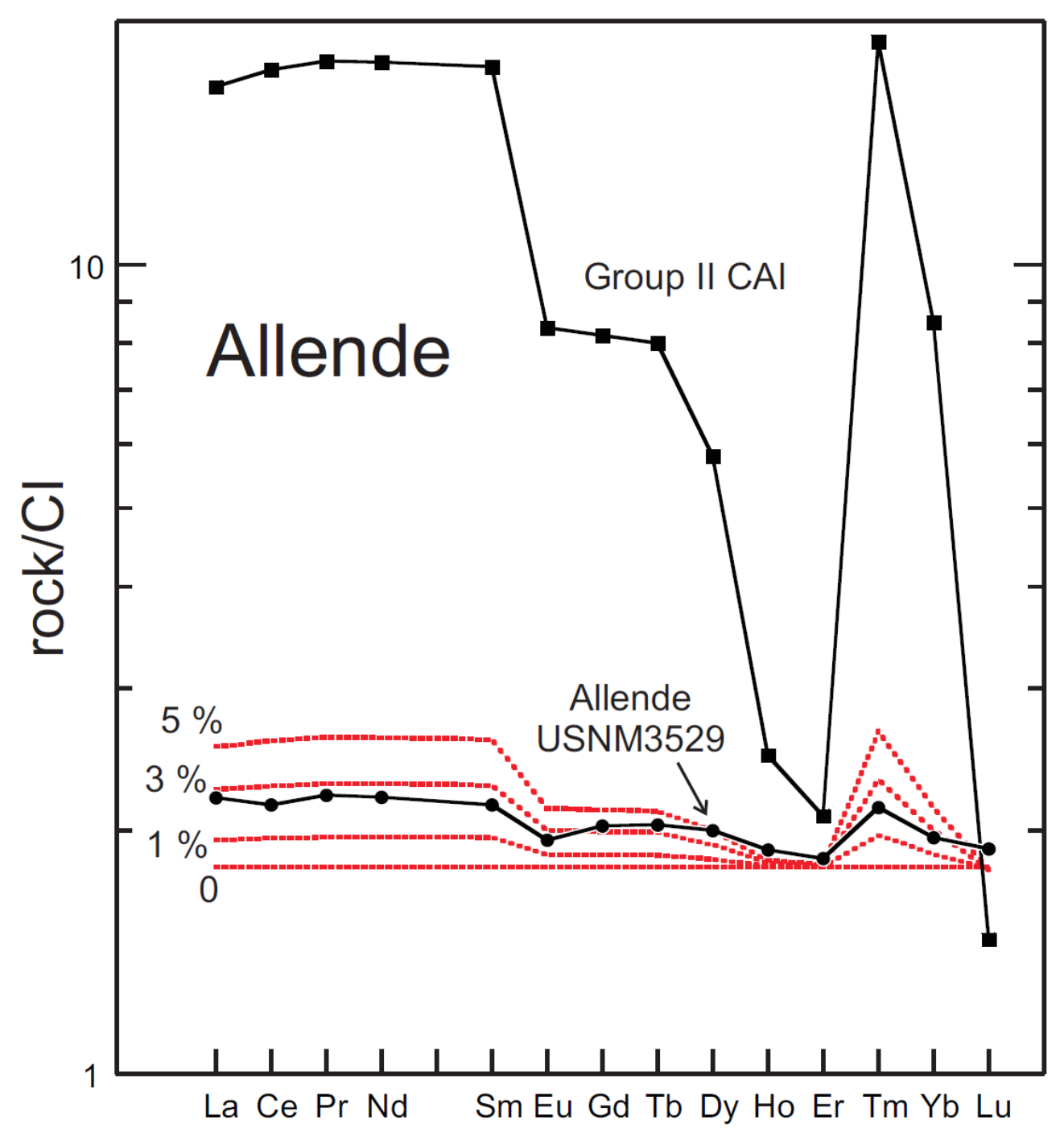

Fig. 6. REE patterns for the Allende CV chondrite (USNM3529, Barrat et al., 2012) and the mean of two group II CAIs obtained by Huang et al. (2012). The REE abundances of this chondrite can be easily reproduced by an addition of 2-3 wt $\%$ of this type of CAIs in a chondritic material with REE concentrations $=1.8 \times \mathrm{CI}$ abundances. 


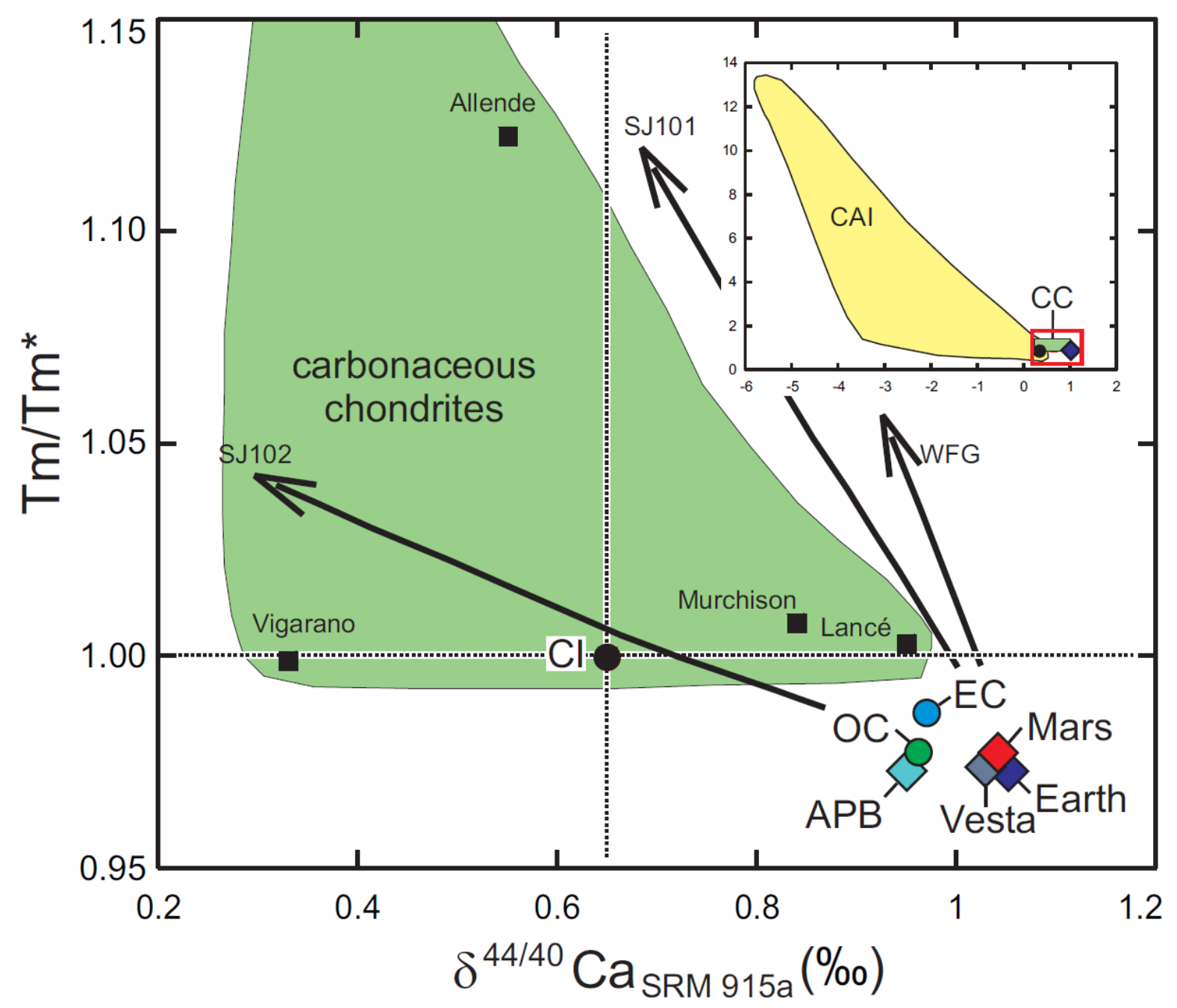

Fig. 7. Relationship between $\mathrm{Tm} / \mathrm{Tm} *$ ratios and $\mathrm{Ca}$ isotopic composition in chondrite groups, Earth, Mars, Vesta and the aubrite parent body (APB). The black arrows indicate mixing between a bulk silicate Earth composition and selected refractory inclusions analyzed by Huang et al. (2012). The labels on the curves correspond to the names of the inclusions used in the calculations. $\mathrm{Tm} / \mathrm{Tm}$ * values for Murchison, Lance, and Vigarano are from Dauphas and Pourmand (2015). Ca isotopic compositions are from Valdes et al. (2014) and Magna et al. (2015). 


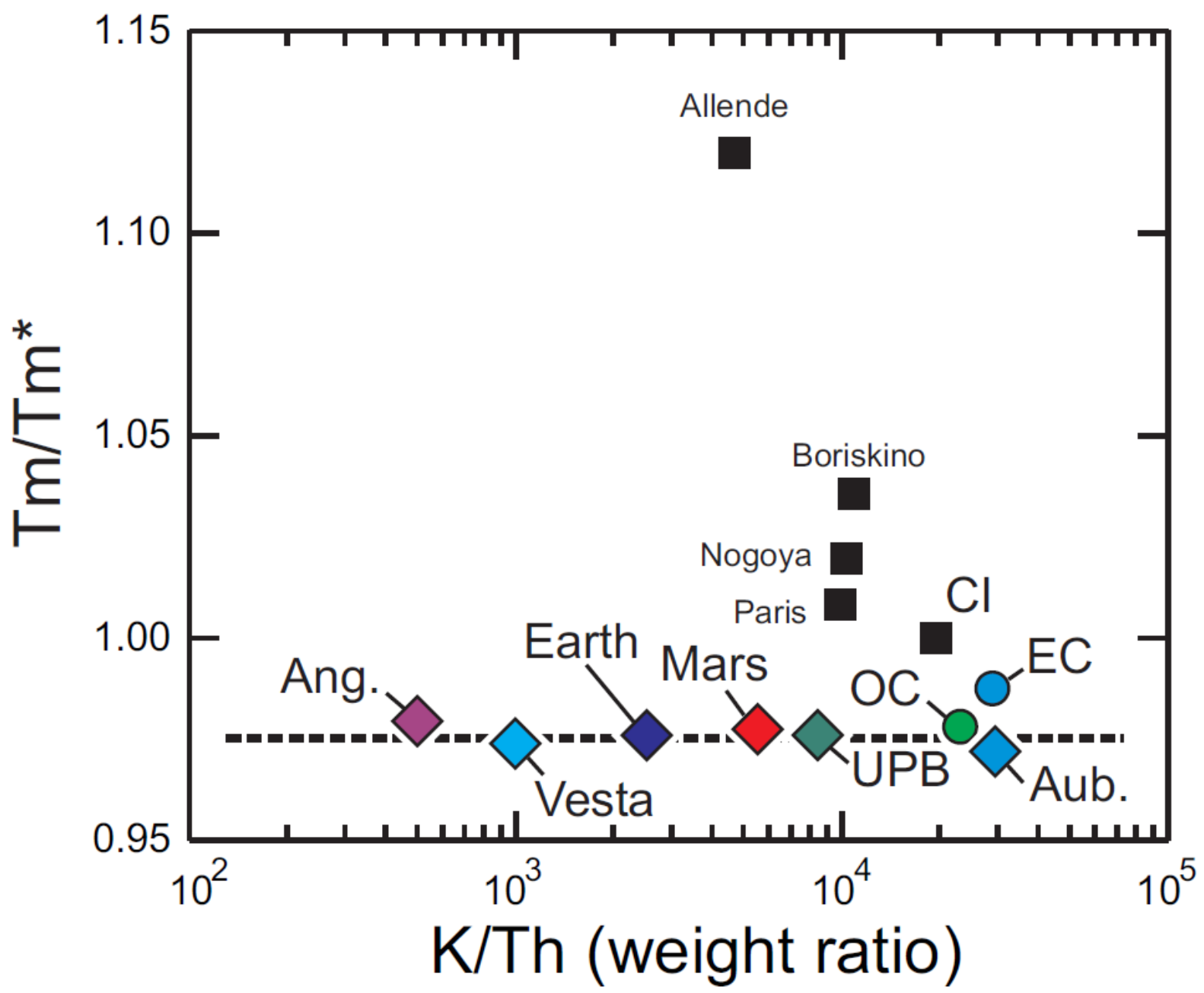

Fig. 8. Plot of $\mathrm{Tm} / \mathrm{Tm} *$ vs. K/Th ratios for bulk differentiated bodies and chondrites. The angrite parent body (Ang.) is inferred from NWA 1296, the sole angrite analyzed in this study. The aubrite parent body (Aub.) and enstatite chondrites are assumed to share the same K/Th ratio. The K/Th ratio of the ureilite parent body (UPB) is inferred from the ALMA trachyandesite (Bischoff et al., 2014). Data for carbonaceous chondrites (black squares) are from Barrat et al. (2012), Hewins et al. (2014) and this study. 